\title{
Avaliação Ultra-sonográfica do Crescimento Fetal em Gestações Gemelares
}

Autora: Mariza Marie Fujita

Orientadora: Dra. Maria Okumura

Tese apresentada à Faculdade de Medicina da Universidade de São Paulo para obtenção do título de Doutor em Medicina, em 15/9/98.

O objetivo deste estudo foi: A) determinar curvas e tabelas de parâmetros biométricos ultrasonográficos (diâmetro biparietal, circunferência cefálica, circunferência abdominal, comprimento do fêmur e peso estimado) em relação à idade gestacional; B) estabelecer um modelo de estimativa da idade gestacional; C) analisar eventuais diferenças entre as curvas obtidas na população em estudo e as curvas existentes para fetos únicos.

Este estudo prospectivo e longitudinal compreendeu 34 gestações gemelares avaliadas em um período de três anos na Clínica Obstétrica do Hospital das Clinicas da Faculdade de Medicina da Universidade de São Paulo. Exames ultrasonográficos fetais seriados foram realizados por um único observador.

Os critérios de inclusão foram: (1) ausência de qualquer patologia materna intercorrente durante a gestação, (2) ausência de malformação ou óbito fetal, (3) primeira ultra-sonografia obstétrica realizada até a $24^{a}$ semana de gestação, (4) ausência de discordância de peso entre os gêmeos e (5) gestações gemelares duplas.

Por meio de modelos de efeitos aleatórios, foram descritas curvas de crescimento longitudinal médio entre 12 e 39 semanas para cada parâmetro biométrico ultra-sonográfico a partir de curvas de crescimento individual.

A combinação de parâmetros fetais apresentou melhor estimativa da idade gestacional do que a utilização de qualquer parâmetro isolado. A estimativa da idade gestacional por meio de modelo polinomial a partir da circunferência cefálica e do comprimento do fêmur produziu resultados equivalentes ao modelo envolvendo quatro parâmetros biométricos.

Comparando-se os valores médios e os respectivos intervalos de confiança $(95 \%)$ de cada parâmetro biométrico ultrasonográfico com os de fetos únicos descritos na literatura, constataramse diferenças principalmente no terceiro trimestre da gestação.

Este estudo sugere que os modelos descritos por Hadlock et al. subestimam a idade de gestações gemelares dessa população no terceiro trimestre.

Palavras-chave: Feto: crescimento e desenvolvimento. Gemeralidade. Gravidez normal.

\section{Correlação dos Aspectos Laparoscópicos com os Achados Histológicos na Endometriose Peritoneal à Luz da Teoria Evolutiva}

Autor: Francesco Antônio Viscomi Orientador: Prof. Dr. Rogério Dias

Tese apresentada ao curso de Pós-Graduação em Ginecologia da Faculdade de Medicina de Botucatu, UNESP, para obtenção do Título de Doutor, em 11/12/98.

A correlação dos aspectos laparoscópicos com os achados histológicos na endometriose peritoneal tem estimulado vários estudos com o objetivo de facilitar a compreensão da teoria evolutiva da endometriose. Neste estudo foi avaliado a correlação dos aspectos laparoscópicos com a história e a profundidade da lesão endometriótica peritoneal à luz da teoria evolutiva da endometriose. Foram selecionadas aleatoriamente para o estudo, 67 pacientes submetidas à laparoscopia, sendo 41 pacientes por algia pélvica, 17 por infertilidade, 5 por tumor anexial e 4 por outras indicações. A idade das pacientes variou entre 15 e 45 anos. Em relação à paridade, $79,1 \%$ eram nulíparas. A avaliação laparoscópica baseou-se no aspecto visual do 